\title{
Ipsilateral ultrasound-monitoring technique for reducing malpositions of peripherally inserted central catheters in the intensive care unit
}

\author{
Seunghwan Song ${ }^{\wedge}$, Up Huh ${ }^{1 \wedge}$, Jae Il Lee ${ }^{2}$, Chung Won Lee ${ }^{1 \wedge}$, Jung Seop Eom³, Hyo-Jeong Kim, \\ Il Jae Wang ${ }^{5}$, Jae-Joon Kim ${ }^{6}$
}

${ }^{1}$ Department of Thoracic and Cardiovascular Surgery, Biomedical Research Institute, Pusan National University Hospital, Pusan National University School of Medicine, Busan, Republic of Korea; ${ }^{2}$ Department of Neurosurgery, Biomedical Research Institute, Pusan National University Hospital, Pusan National University School of Medicine, Busan, Republic of Korea; ${ }^{3}$ Division of Respiratory, Department of Internal Medicine, Biomedical Research Institute, Pusan National University Hospital, Pusan National University School of Medicine, Busan, Republic of Korea; ${ }^{4}$ Division of Hematology-Oncology, Department of Internal Medicine, Medical Research Institute, Pusan National University Hospital, School of Medicine, Busan, Republic of Korea; ${ }^{5}$ Department of Emergency Medicine, Biomedical Research Institute, Pusan National University Hospital, Pusan National University School of Medicine, Busan, Republic of Korea; ${ }^{6}$ Division of Hematology-Oncology, Department of Internal Medicine, Medical Research Institute, Pusan National University Yangsan Hospital, School of Medicine, Yangsan, Republic of Korea

Contributions: (I) Conception and design: All authors; (II) Administrative support: CW Lee; (III) Provision of study materials or patients: JI Lee, JS Eom, HJ Kim; (IV) Collection and assembly of data: S Song, U Huh; (V) Data analysis and interpretation: U Huh, IJ Wang, JJ Kim; (VI) Manuscript writing: All authors; (VII) Final approval of manuscript: All authors.

Correspondence to: Up Huh, MD. Department of Thoracic and Cardiovascular Surgery, Biomedical Research Institute, Pusan National University Hospital, Pusan National University School of Medicine, 179 Gudeok-Ro, Seo-Gu, Busan 49241, Republic of Korea. Email: tymfoo82@gmail.com.

Backgrounda We performed ultrasonographic examinations of the ipsilateral internal jugular vein after placement of peripherally inserted central catheters (PICCs) and found that more than half of malpositions of PICC went to the head through the ipsilateral internal jugular vein. We aimed to reduce malpositions of PICCs in settings where fluoroscopy is not available.

Methods: This retrospective study had a case-control, single-center study design. From January to June 2018, PICCs were inserted with the patient's arm abducted without the use of ultrasonography to identify the presence of the PICC in the ipsilateral internal jugular vein (procedure A). From July to December 2018, PICCs were inserted in a similar fashion; however, ultrasonography was employed to detect the catheter tip in the ipsilateral internal jugular vein (procedure B).

Results: There were 794 placements of PICCs in 650 patients. Procedures A and B were performed in 418 and 376 patients, respectively. Malpositioning of PICC tips occurred significantly less often in procedure B than in procedure $\mathrm{A}(2.4 \%$ vs. $5.7 \%, \mathrm{P}=0.02)$.

Conclusions: In a setting without fluoroscopy, insertions of PICCs can be performed with the arm abducted at $90^{\circ}$. Ultrasound monitoring for the presence of the catheter in the ipsilateral internal jugular vein can help with correct positioning, thereby reducing the occurrence of catheter tip malpositions.

Keywords: Malposition; peripherally inserted central catheter (PICC); ultrasound

Submitted May 30, 2020. Accepted for publication Sep 18, 2020.

doi: 10.21037/apm-20-1201

View this article at: http://dx.doi.org/10.21037/apm-20-1201

^ ORCID: Seunghwan Song, 0000-0002-7492-2097; Up Huh, 0000-0002-7739-3979; Chung Won Lee, 0000-0002-1160-6003. 


\section{Introduction}

A peripherally inserted central venous catheter (PICC) is inserted via a peripheral vein, with the tip residing in a central vein (1). PICCs are widely used in the intensive care unit (ICU) for mid- and long-term central venous access (2). Compared with the traditional central venous catheter (CVC), a PICC inserted into the upper arm is safer and has a lower infection rate and a longer duration of use $(3,4)$.

In general, an interventional radiologist places a PICC under fluoroscopic guidance. However, most patients in the ICU are unable to move to the interventional radiology suite because of respiratory failure or hemodynamic instability. In these cases, which require PICC placement in the ICU, fluoroscopy cannot be used, and the position of the catheter tip must be confirmed using radiography after insertion. This also monitors the catheter movement into the internal jugular vein (IJV) after insertion. A CVC tip located at or above the level of the upper superior vena cava (SVC) is more likely associated with thrombosis and an increased risk of migration $(5,6)$.

In this study, more than half of PICC malpositions went to the head through the IJV. We performed ultrasonographic examinations of the IJV after PICC insertion to indirectly confirm whether the catheter was present and to compare these findings with the previous radiographic confirmations after PICC placement.

We present the following article in accordance with the STROBE Statement reporting checklist (available at http:// dx.doi.org/10.21037/apm-20-1201).

\section{Methods}

We conducted a retrospective, case-control, single-center study of patients aged 18-95 years who required a PICC in the ICU of a single institution from January 1, 2018, to December 31, 2018. All PICC procedures in the ICU took place at the bedside and were performed in the upper arm by a single vascular surgeon.

From January to June 2018, PICCs were inserted with the arm abducted at $90^{\circ}$ from the patient's body and the head turned toward the ipsilateral side (if possible) under ultrasonic guidance. The presence of the PICC in the ipsilateral IJV was not confirmed using ultrasonography during this timeframe (procedure A). From July to December 2018, PICCs were inserted with the same posture as in procedure $\mathrm{A}$. This time, however, the presence of the PICC in the ipsilateral IJV was confirmed using ultrasonography (procedure B) to compare the accuracy of the PICC tip positioning using the two methods. The same LOGIQ e ultrasound (GE Healthcare, Chicago, IL, USA) was used for both PICC procedure and ipsilateral IJV monitoring.

The catheter tip position ideally rests at the junction of the SVC and the right atrium, also called the cavoatrial junction (CAJ). The CAJ was defined to be $5 \mathrm{~cm}$ below the right tracheobronchial angle, based on the reported median distance of $4.9 \mathrm{~cm}$ (range, 2.9-6.8 $\mathrm{cm}$ ) from the right tracheobronchial angle to the CAJ (7). Catheter tip position was graded according to the following classification scheme: 0 indicated containment within the body of one thoracic spine containing the junction; -1 signified one spine body cephalad to the thoracic spine containing the junction; -2 denoted greater than the body of the thoracic spine containing the junction, at two spine bodies cephalad; and +1 was defined as one spine body caudal to the body of the thoracic spine, including the junction (Figure 1).

\section{Operative procedure}

One cardiovascular surgeon performed all PICC insertions (Figure 2). The surgeon used a 5-Fr dual-lumen Xcela ${ }^{\circledR}$ Power Injectable PICC (Navilyst Medical Inc., MA, USA) or a 6-Fr triple-lumen Pro-PICC CT Injectable Catheter (Medcomp, PA, USA). Maximal barrier precautions, including cap, mask, sterile gown, sterile gloves, and vast sterile field, were consistently used during the procedures. Peripheral venous access was obtained through the basilic or brachial veins with ultrasound guidance. A standard 5- to $12-\mathrm{MHz}$ linear ultrasound probe was used. Lidocaine (2\%, $1-2 \mathrm{~mL}$ ) was injected into the subcutaneous tissue for local anesthesia.

Under ultrasound guidance, the target vessel was punctured using a 20-gauge needle, and blood return in the needle was confirmed. A 0.018-inch guide wire was then introduced into the vein blindly. The puncture site was then enlarged slightly with a scalpel blade, and a 5-Fr microintroducer assembly was introduced over the guidewire. The length of the PICC was determined as the distance from the puncture site to the humeral head, sternal notch, and third intercostal space. The catheter was inserted into the micro-introducer sheath. In procedure $\mathrm{B}$, the presence of the catheter tip in the ipsilateral IJV was immediately checked using ultrasonography. When the catheter was seen on the sonogram, it was pulled back sufficiently and repeatedly positioned until no part of the catheter 


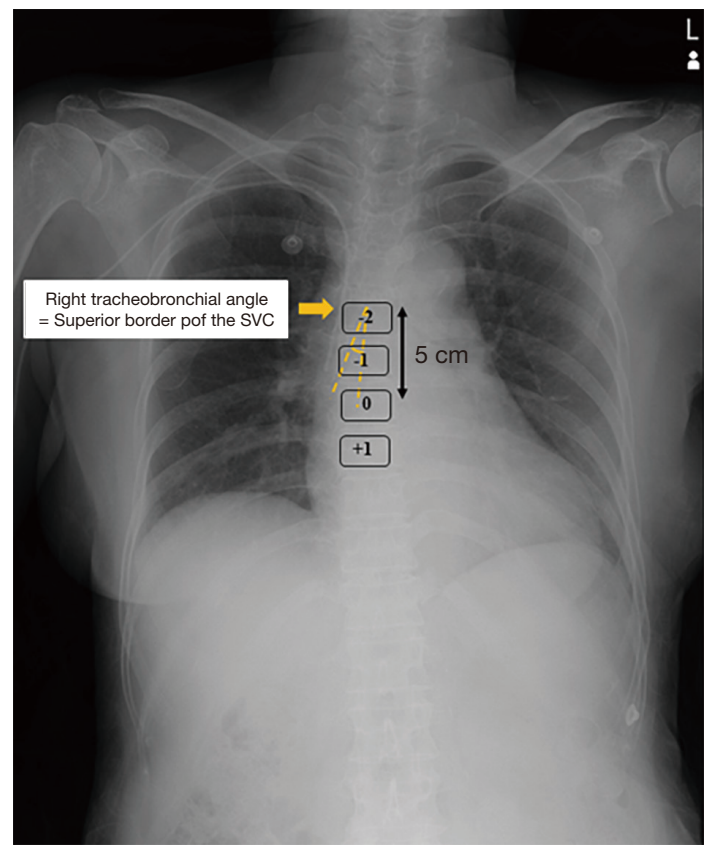

Figure 1 Definitions of peripherally inserted central catheter tip positions. The catheter tip position was based on the junction of the superior vena cava (SVC) and right atrium, also called the cavoatrial junction (CAJ). The CAJ was defined as the site $5 \mathrm{~cm}$ below the right tracheobronchial angle. 0: the catheter tip was contained within the body of one thoracic spine containing the junction; -1 : the catheter tip was located in one spine body cephalad to the thoracic spine containing the junction; -2 : the location of the catheter tip was greater than the body of the thoracic spine containing the junction, at two spine bodies cephalad; +1: the catheter tip was located in one spine body caudal to the body of the thoracic spine including the junction.

was visible on sonogram (Figure 3). After completion of procedures $\mathrm{A}$ and $\mathrm{B}$, a chest radiograph was obtained to confirm the position of the catheter tip. A malposition was defined as a catheter tip location in the innominate vein or IJV and not in the SVC (Figure 4). The dressing was changed every 7 days or when soiled.

\section{Statistical analysis}

A two-sample $t$-test or Wilcoxon rank-sum test was used for continuous variables, and Fisher's exact test was used for categorical variables. All analyses were performed using SAS version 9.3 (SAS Institute., Cary, NC, USA), and a $\mathrm{P}<0.05$ was considered statistically significant.

\section{Ethical statement}

The study was conducted in accordance with the Declaration of Helsinki (as revised in 2013). The study was approved by the institutional review board from the Pusan National University Hospital (H-1909-017-083), and individual consent for this retrospective analysis was waived.

\section{Results}

From January 2018 to December 2018, there were 794 PICC placements in 650 patients. Procedures A and B were performed on 418 and 376 patients, respectively. The characteristics of the two groups are described in Table 1. PICC malpositions were identified by chest radiography in $5.7 \%$ and $2.4 \%$ of patients after procedures $\mathrm{A}$ and $\mathrm{B}$, respectively. There were significantly less malpositions during procedure $\mathrm{B}(\mathrm{P}=0.02)$. A significant difference was found in the catheter tip location between the two groups $(\mathrm{P}=0.02)$. When the arm was abducted at $90^{\circ}$, it seemed to be more accurate to manually determine the length of the PICC. Table 2 shows the results of the classification based on the location of the malposition, as shown in Figure 4. Malposition B, with the catheter tip located in the IJV, occurred less often in procedure $B$ than in procedure $A$; however, there were no statistically significant differences. The outcomes associated with PICC insertions are 

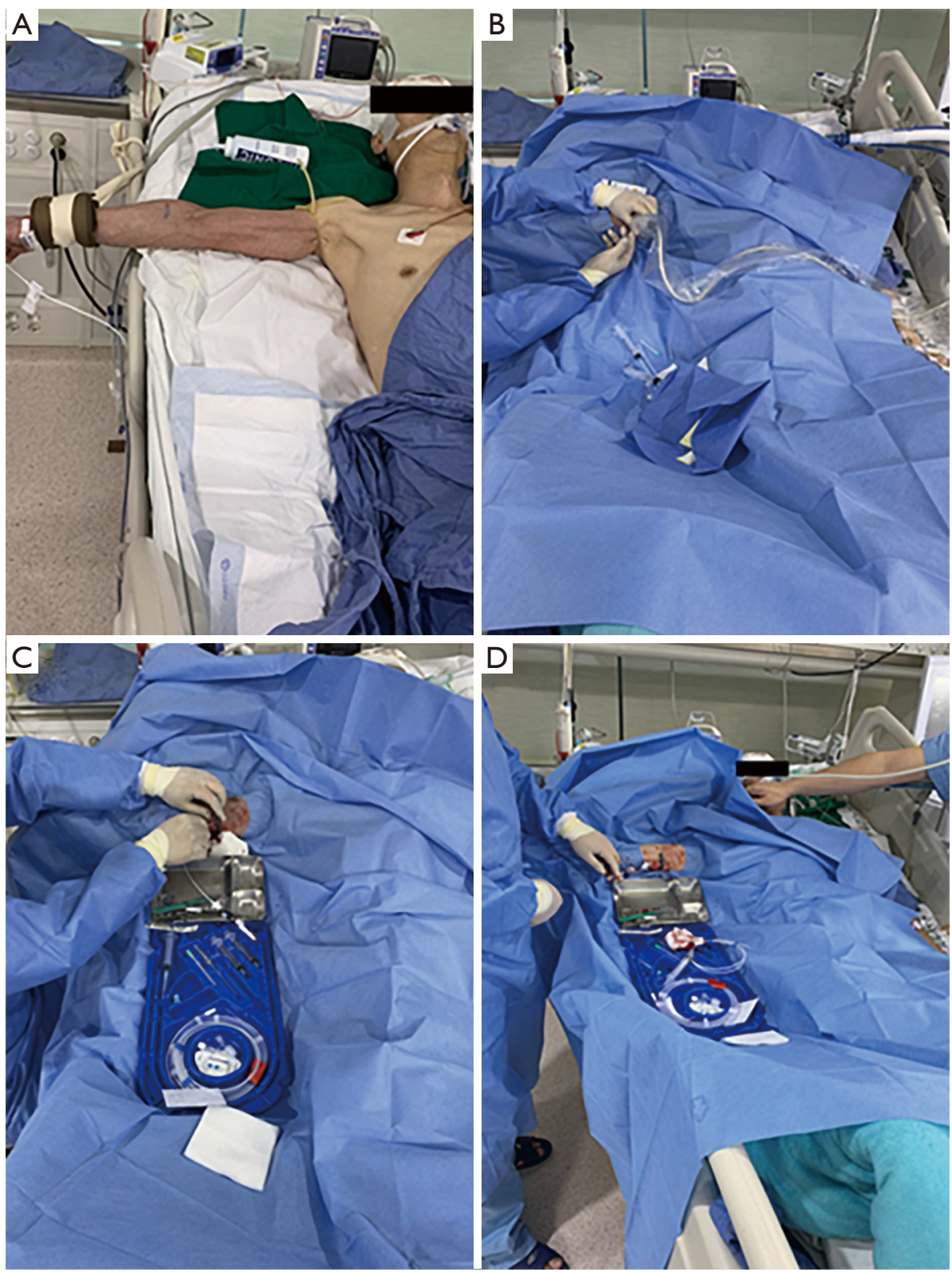

Figure 2 Procedure B. (A) The peripherally inserted central catheter (PICC) was inserted with the arm abducted $90^{\circ}$ from the patient's body with the head turned toward the ipsilateral side (if possible); (B) maximal barrier precautions were consistently used during the procedure, including a cap, mask, sterile gown, sterile gloves, and vast sterile field. Under ultrasound guidance, the target vessel was punctured with a 20-gauge needle and blood return was confirmed; (C) the catheter was inserted into the micro-introducer sheath; (D) the position of the catheter tip in the ipsilateral internal jugular vein was immediately checked using ultrasonography.

described in Table 3. The causes of PICC removals were also examined. There were no significant differences between the two groups based on discharge, death, infection, malfunction, or self-removal.

\section{Discussion}

CVC placement is a routine central venous access procedure performed in the ICU setting for administration of drugs, including vasopressors, inotropes, antibiotics, 

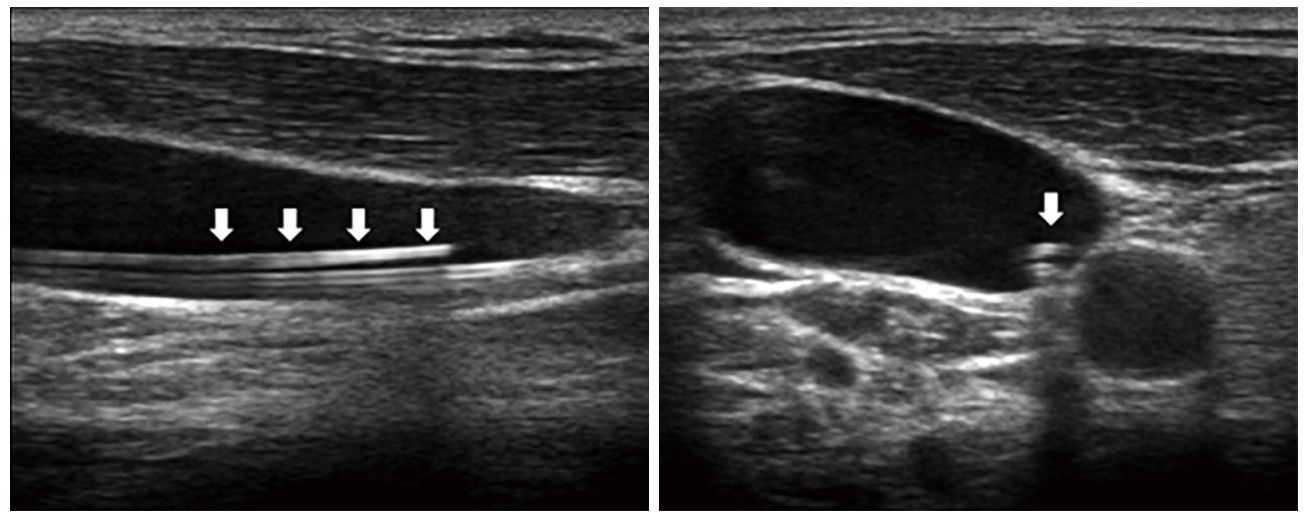

Figure 3 Peripherally inserted central catheter (PICC) tip in the internal jugular vein. The presence of a PICC catheter (white arrows) in the internal jugular vein can be confirmed using ultrasonography.
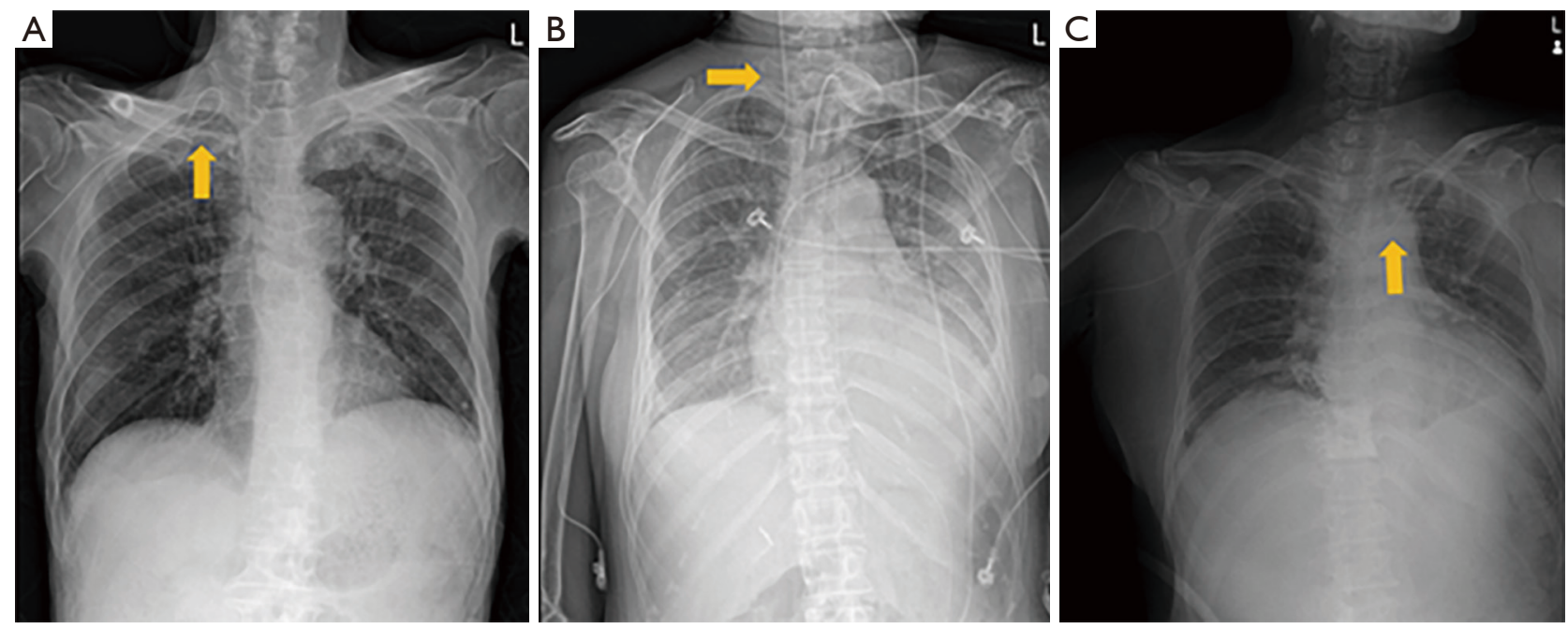

Figure 4 Examples of peripherally inserted central catheter (PICC) malpositions. (A) The catheter tip is located in the right subclavian vein (arrow); (B) the catheter tip is located in the right internal jugular vein (arrow); (C) the catheter tip is located in the left innominate (brachiocephalic) vein (arrow).

or chemotherapy, central venous pressure monitoring, central venous oxygen saturation measurements, and total parenteral nutrition. Unlike other types of central venous access, PICC is increasingly being used for its ease of placement and its low rate of insertion-related mechanical complications, including venous thromboses and infections (8-12). Although a PICC is inserted to maintain central venous access in patients admitted to the ICU, it is not easy to correctly position the catheter without using fluoroscopy.

The bedside ultrasonography and saline flush technique have both been identified as methods to reduce PICC malpositions in settings where fluoroscopy is not available, such as in the ICU $(13,14)$. Despite its proven usefulness, the saline flush technique requires additional heart ultrasound probes and a professional assistant to identify saline bubbles in the right atrium using ultrasonography. In a meta-analysis conducted by Bou Chebl et al. (14), contrast-enhanced ultrasonography showed a pooled sensitivity of $72 \%$, pooled specificity of $100 \%$ (95\% CI, 99-100\%), positive predictive value of $92.1 \%$, and negative predictive value of $98.5 \%$ compared to chest radiography for confirming CVC placement.

Li et al. used an electrocardiograph (ECG)-guided PICC tip location technique to confirm the catheter tip 
Table 1 Comparison of procedure A and procedure B

\begin{tabular}{|c|c|c|c|c|}
\hline Variables & Total $(n=794)$ & Procedure $A^{\dagger}(n=418,52.6 \%)$ & Procedure $B^{\ddagger}(n=376,47.4 \%)$ & $P$ value \\
\hline Male, n (\%) & $450(56.7)$ & $232(55.5)$ & $218(58.0)$ & 0.52 \\
\hline Survival, n (\%) & $633(79.7)$ & $325(77.8)$ & $308(81.9)$ & 0.16 \\
\hline Insertion in the right arm, $\mathrm{n}(\%)$ & $640(80.6)$ & 344 (82.3) & $296(78.7)$ & 0.21 \\
\hline Catheter tip position", n (\%) & & & & $0.02^{*}$ \\
\hline-2 & $2(0.3)$ & $2(0.5)$ & $0(0.0)$ & \\
\hline-1 & $26(3.3)$ & $19(4.5)$ & $7(1.9)$ & \\
\hline 0 & $639(80.5)$ & $323(77.3)$ & $316(84.0)$ & \\
\hline \multicolumn{5}{|c|}{$\begin{array}{l}\text { ', from January to June } 2018 \text {, peripherally inserted central catheters (PICCs) were inserted with the arm adducted under ultrasound } \\
\text { guidance. We did not confirm the presence of the PICC in the ipsilateral internal jugular vein (IJV) using ultrasonography during this } \\
\text { timeframe. }{ }^{\ddagger} \text {, from July to December } 2018 \text {, PICCs were inserted with the arm abducted } 90^{\circ} \text { under ultrasound guidance. We confirmed the } \\
\text { presence of the PICC catheter in the ipsilateral IJV using ultrasonography. }{ }^{\S} \text {, when the tip of the catheter was not located in the superior } \\
\text { vena cava (SVC) but was located in the innominate vein or IJV. ", catheter tip position was based on the cavoatrial junction, defined as the } \\
\text { site } 5 \mathrm{~cm} \text { below the right tracheobronchial angle. }{ }^{*} \text {, significant P values. } 0 \text { : the catheter tip was contained within the body of one thoracic } \\
\text { spine containing the junction; }-1 \text { : the catheter tip was located in one spine body cephalad to the thoracic spine containing the junction; } \\
-2 \text { : the location of the catheter tip was greater than the body of the thoracic spine containing the junction, at two spine bodies cephalad; } \\
+1 \text { : the catheter tip was located in one spine body caudal to the body of the thoracic spine including the junction. }\end{array}$} \\
\hline
\end{tabular}

Table 2 Classification by malposition location

\begin{tabular}{lcc}
\hline Malposition & Procedure $A^{\dagger}(n=24)$ & Procedure $B^{\ddagger}(n=9)$ \\
\hline$A^{\S}$ & $2(8.3)$ & $1(11.1)$ \\
$\mathrm{B}^{\Uparrow \Uparrow}$ & $14(58.3)$ & $2(22.2)$ \\
$\mathrm{C}^{\dagger \dagger}$ & $8(33.3)$ & $6(66.7)$ \\
\hline
\end{tabular}

${ }^{\dagger}$, from January to June 2018, peripherally inserted central catheters (PICCs) were inserted with the arm adducted under ultrasound guidance. We did not confirm the presence of the PICC in the ipsilateral internal jugular vein (IJV) using ultrasonography at this time. ${ }^{\ddagger}$, from July to December 2018, PICCs were inserted with the arm abducted $90^{\circ}$ under ultrasound guidance. We confirmed the presence of the PICC catheter in the ipsilateral IJV using ultrasonography. ${ }^{\S}$, the catheter tip is located in the right subclavian vein. ", the catheter tip is located in the right internal jugular vein. ${ }^{\dagger \dagger}$, the catheter tip is located in the opposite left innominate (brachiocephalic) vein (see Figure 4).

location (15). When the catheter was inserted almost up to the estimated length, the right arm lead was removed, and the clamps were sterilized and attached to the guide wire. The guide wire was then advanced slowly, 0.5 to $1 \mathrm{~cm}$ at a time, while observing the $\mathrm{P}$-wave changes on the monitor. The ECG test group (99.3\%) achieved a significantly more accurate tip placement than did the control group $(92.3 \%$, $\mathrm{P}<0.001$ ); however, this method cannot be used in patients with heart disease or arrhythmia and can present risks of contamination (16).
In the present study, malpositions were reduced by half with procedure B $(2.4 \%)$ compared with procedure A $(5.7 \%)$ because ultrasonography successfully identified malpositions from the IJV upwards. This finding was observed because ultrasonography identified malpositions from the IJV upwards (Figure 4B). As shown in Table 2, 14 cases of malposition B occurred in procedure A and two cases in procedure B. Even though we observed the IJV using ultrasonography, we missed two cases. It is presumed that despite the monitoring of the IJV using ultrasonography, 
Table 3 Outcomes of PICC placements

\begin{tabular}{|c|c|c|c|c|}
\hline Cause of removal & Total $(n=794)$ & Procedure $\mathrm{A}^{\dagger}(\mathrm{n}=418,52.6 \%)$ & Procedure $B^{\ddagger}(n=376,47.4 \%)$ & $P$ value \\
\hline Death, n (\%) & $129(16.2)$ & $70(16.7)$ & $59(15.7)$ & 0.70 \\
\hline Infection, n (\%) & $112(14.1)$ & $68(16.3)$ & $44(11.7)$ & 0.07 \\
\hline Malfunction, n (\%) & $10(1.3)$ & $5(1.2)$ & $5(1.3)$ & 1.00 \\
\hline Arm swelling, oozing, or pain, $\mathrm{n}(\%)$ & $16(2.0)$ & $8(1.9)$ & $8(2.1)$ & 1.00 \\
\hline
\end{tabular}

${ }^{\dagger}$, from January to June 2018, peripherally inserted central catheters (PICCs) were inserted with the arm adducted under ultrasound guidance. We did not confirm the presence of the PICC in the ipsilateral internal jugular vein (IJV) using ultrasonography at this time. ${ }^{\ddagger}$, from July to December 2018, PICCs were inserted with the arm abducted $90^{\circ}$ under ultrasound guidance. We confirmed the presence of PICC catheter in the ipsilateral IJV using ultrasonography. *, significant $\mathrm{P}$ value.

these cases were missed because of the insufficient monitoring to the proximal portion of the IJV. Malpositions $\mathrm{A}$ and $\mathrm{C}$ could not be resolved by ultrasound observation of the IJV because the catheter tip was located in the right subclavian vein and left innominate vein. Although the number of malposition B cases decreased significantly, malposition of $2.4 \%$ occurred.

According to Schweickert et al., when monitoring the ipsilateral IJV while placing PICCs at the bedside, the malposition of the ipsilateral IJV was significantly lower than that of the unmonitored ipsilateral IJV $(0.7 \% v s$. $7.4 \%, \mathrm{P}=0.003)$. The number of cases where the PICC was accurately located in the SVC was statistically significantly higher in the ipsilateral IJV-monitored group (68.9\% vs. $58.4 \%, \mathrm{P}=0.06)$. In addition, malposition to other proximal vein (subclavian vein, brachiocephalic vein, and axillary vein) occurred in this study, and no statistically significant difference was found between ipsilateral IJV monitoring and non-monitoring (17).

In this study, the accuracy of the catheter tip location was similar to that of the bedside ultrasonography and saline flush technique and an ECG-guided PICC tip location technique and was more accurate than other studies that monitored the ipsilateral IJV using ultrasonography; however, the technique for ultrasound monitoring of the ipsilateral IJV did not require a professional assistant nor an echocardiography probe, and patients with arrhythmias or heart disease could safely go through this procedure.

In both procedures, chest radiographs were essential for determining the catheter tip positions. Nevertheless, PICC placement continues to be a challenge in the context of venospasm, venous tortuosity, and venous valves (1).
The limitation of this method is that it cannot identify malpositions, as illustrated in Figure 3A,C.

Most guidelines recommend that the catheter tip should be located in the inferior third of the SVC at the junction of the right atrium; however, the ideal location for the CVC tip is still a matter of debate (18-20). The right tracheobronchial angle is considered the best landmark for catheter tip positioning (20) and the most reliable radiographic landmark to define the borders of the SVC. This angle is created as the right main bronchus bifurcates from the trachea. The superior border of the SVC is always superior to the right tracheobronchial angle $(7,21)$. The median distance from the right tracheobronchial angle to the CAJ is $4.9 \mathrm{~cm}$ (range, 2.9-6.8 cm) (7). We found that procedure $\mathrm{B}$ allowed for significantly more accurate positioning of the catheter tip than procedure A. Nevertheless, the effect of increasing operator experience over time as a possible bias between the methods cannot be ruled out.

There were some limitations to this study. There was a limitation in statistical analysis because this study was not a randomized trial, and both groups were not conducted simultaneously. In addition, all procedures were performed by one operator. Nevertheless, there have been no previous studies confirming PICC placement in the ipsilateral IJV using ultrasonography to reduce malpositions in settings where fluoroscopy is not available. This technique was simple and easy to perform, did not require echocardiography probes or trained assistants, could be performed in patients with or without arrhythmias or heart diseases, and had a similar accuracy compared to previous methods. In the future, a method to monitor the location of 
the catheter tip in real time and without an interventional suite should be developed.

\section{Acknowledgments}

Funding: None.

\section{Footnote}

Reporting Checklist: The authors have completed the STROBE reporting checklist. Available at http://dx.doi. org/10.21037/apm-20-1201

Data Sharing Statement: Available at http://dx.doi. org/10.21037/apm-20-1201

Peer Review File: Available at http://dx.doi.org/10.21037/ apm-20-1201

Conflicts of Interest: All authors have completed the ICMJE uniform disclosure form (available at http://dx.doi. org/10.21037/apm-20-1201). The authors have no conflicts of interest to declare.

Ethical Statement: The authors are accountable for all aspects of the work in ensuring that questions related to the accuracy or integrity of any part of the work are appropriately investigated and resolved. The study was conducted in accordance with the Declaration of Helsinki (as revised in 2013). The study was approved by the institutional review board from the Pusan National University Hospital (H-1909-017-083), and individual consent for this retrospective analysis was waived.

Open Access Statement: This is an Open Access article distributed in accordance with the Creative Commons Attribution-NonCommercial-NoDerivs 4.0 International License (CC BY-NC-ND 4.0), which permits the noncommercial replication and distribution of the article with the strict proviso that no changes or edits are made and the original work is properly cited (including links to both the formal publication through the relevant DOI and the license). See: https://creativecommons.org/licenses/by-nc-nd/4.0/.

\section{References}

1 Racadio JM, Doellman DA, Johnson ND, et al. Pediatric peripherally inserted central catheters: complication rates related to catheter tip location. Pediatrics 2001;107:E28.

2 Marcy PY. Central venous access: techniques and indications in oncology. Eur Radiol 2008;18:2333-44.

3 Morano SG, Latagliata R, Girmenia C, et al. Catheterassociated bloodstream infections and thrombotic risk in hematologic patients with peripherally inserted central catheters (PICC). Support Care Cancer 2015;23:3289-95.

4 Sakai T, Kohda K, Konuma Y, et al. A role for peripherally inserted central venous catheters in the prevention of catheter-related blood stream infections in patients with hematological malignancies. Int J Hematol 2014;100:592-8.

5 Waghorn DJ. Intravascular device-associated systemic infections: a 2 year analysis of cases in a district general hospital. J Hosp Infect 1994;28:91-101.

6 Fletcher SJ, Bodenham AR. Safe placement of central venous catheters: where should the tip of the catheter lie? Br J Anaesth 2000;85:188-91.

7 Aslamy Z, Dewald CL, Heffner JE. MRI of central venous anatomy: implications for central venous catheter insertion. Chest 1998;114:820-6.

8 Wilson TJ, Stetler WR Jr, Fletcher JJ. Comparison of catheter-related large vein thrombosis in centrally inserted versus peripherally inserted central venous lines in the neurological intensive care unit. Clin Neurol Neurosurg 2013;115:879-82.

9 Potet J, Arnaud FX, Thome A, et al. Peripherally inserted central catheter placement in patients with coagulation disorders: a retrospective analysis. Diagn Interv Imaging 2015;96:1147-51.

10 Pittiruti M, Hamilton H, Biffi R, et al. ESPEN guidelines on parenteral nutrition: central venous catheters (access, care, diagnosis and therapy of complications). Clin Nutr 2009;28:365-77.

11 Pittiruti M, Brutti A, Celentano D, et al. Clinical experience with power-injectable PICCs in intensive care patients. Crit Care 2012;16:R21.

12 Wilson TJ, Brown DL, Meurer WJ, et al. Risk factors associated with peripherally inserted central venous catheter-related large vein thrombosis in neurological intensive care patients. Intensive Care Med 2012;38:272-8.

13 Yesilbas O, Sevketoglu E, Kihtir HS, et al. Use of bedside ultrasonography and saline flush technique for evaluation of central venous catheter placement in children. Artif Organs 2018;42:1157-63.

14 Bou Chebl R, Kiblawi S, El Khuri C, et al. Use of contrastenhanced ultrasound for confirmation of central venous catheter placement: systematic review and meta-analysis. J 
Ultrasound Med 2017;36:2503-10.

15 Li A, Jiao J, Zhang Y, et al. A randomized controlled study of bedside electrocardiograph-guided tip location technique \& the traditional chest radiography tip location technique for peripherally inserted central venous catheter in cancer patients. Indian J Med Res 2018;147:477-83.

16 Zhao R, Chen C, Jin J, et al. Clinical evaluation of the use of an intracardiac electrocardiogram to guide the tip positioning of peripherally inserted central catheters. Int J Nurs Pract 2016;22:217-23.

17 Schweickert WD, Herlitz J, Pohlman AS, et al. A randomized, controlled trial evaluating postinsertion neck ultrasound in peripherally inserted central catheter procedures. Crit Care Med 2009;37:1217-21.

Cite this article as: Song S, Huh U, Lee JI, Lee CW, Eom JS, Kim HJ, Wang IJ, Kim JJ. Ipsilateral ultrasound-monitoring technique for reducing malpositions of peripherally inserted central catheters in the intensive care unit. Ann Palliat Med 2021;10(2):1530-1538. doi: 10.21037/apm-20-1201
18 Dulce M, Steffen IG, Preuss A, et al. Topographic analysis and evaluation of anatomical landmarks for placement of central venous catheters based on conventional chest $\mathrm{x}$-ray and computed tomography. Br J Anaesth 2014;112:265-71.

19 Frykholm P, Pikwer A, Hammarskjöld F, et al. Clinical guidelines on central venous catheterization. Swedish Society of Anaesthesiology and Intensive Care Medicine. Acta Anaesthesiol Scand 2014;58:508-24.

20 Vesely TM. Central venous catheter tip position: a continuing controversy. J Vasc Interv Radiol 2003;14:527-34.

21 Rutherford JS, Merry AF, Occleshaw CJ. Depth of central venous catheterization: an audit of practice in a cardiac surgical unit. Anaesth Intensive Care 1994;22:267-71. 